\title{
¿Afectan las complicaciones perinatales la respuesta sensible de las madres hacia sus hijos/as de 12 meses?*
}

\section{Do adverse pregnancy outcome affect mothers' sensitive response to their 12 month children?}

\author{
Emilia Barceló \\ Jacinta Pérez-Cotapos \\ Cristóbal Baraona \\ María Ignacia Gómez \\ Marcia Olhaberry \\ Chamarrita Farkas* \\ Pontificia Universidad Católica de Chile \\ (Rec.: junio de 2015 - Acept.: abril de 2016)
}

\begin{abstract}
Resumen
La respuesta sensible es una competencia materna ampliamente estudiada por su influencia en el desarrollo infantil y su asociación con las características de la madre, pero en menor medida se ha investigado su relación con las dificultades durante la gestación y el parto. El presente estudio busca identificar la influencia de las complicaciones perinatales sobre la sensibilidad materna en 90 diadas madre-hijo/a entre diez y catorce meses de edad de nivel socioeconómico alto, medio y bajo, residentes en Santiago de Chile. Se aplicó un cuestionario sociodemográfico y las diadas fueron filmadas en una situación de juego libre, la cual fue codificada con la Escala de Sensibilidad del Adulto (E.S.A.). Los resultados muestran que la ausencia de complicaciones en el embarazo de la madre y la presencia de complicaciones en el parto, explican una mayor sensibilidad materna un año después. Se observa, además, una influencia significativa del nivel socioeconómico sobre la sensibilidad materna. Se discuten estos resultados y sus implicancias.
\end{abstract}

Palabras clave: sensibilidad materna, primera infancia, complicaciones perinatales, diadas madre-hijo/a, nivel socioeconómico.

\begin{abstract}
Sensitive response is a maternal competence widely studied for its influence in child development, and for its relationship with mother features, however, there is fewer research on its relationship to the difficulties of pregnancy and childbirth. This study seeks to identify the influence of adverse pregnancy outcome on maternal sensitivity in 90 dyads mother-child between ten and fourteen months age, of high, medium and low socioeconomic status, residents in Santiago, Chile. A sociodemographic questionnaire was taken to mothers, and dyads were filmed in a free play situation, which was coded with the Sensitivity Adult Scale (ESA). Results show that absence of difficulties during pregnancy on the mother, as well as presence of them during labor, explain a higher maternal sensitivity one year later. In addition, a significant influence of socioeconomic status on maternal sensitivity had been observed. These results and their implications are discussed.
\end{abstract}

Keywords: maternal sensitivity, early infancy, adverse pregnancy outcome, mother-child dyads,

socioeconomic status.

\footnotetext{
* Artículo de investigación que contó con la financiación otorgada por el Fondo Nacional de Desarrollo Científico y Tecnológico, proyectos FONDECYT № 1110087 y № 1141118.

** Correspondenca a: Chamarrita Farkas Klein. Escuela de Psicología UC, Avenida Vicuña Mackenna 4860, Macul, Santiago, Chile. E-mail: chfarkas@uc.cl
} 


\section{Introducción}

La teoría del apego desarrollada por John Bowlby (1969) explica la necesidad humana de formar vínculos afectivos estrechos a temprana edad, siendo la base de lo propuesto los conceptos de "reciprocidad en la relación", y la "sensibilidad del adulto frente a las necesidades del niño". Tanto el cuidador como el bebé forman parte de la díada afectiva en que la calidad de sus interacciones constituye una precondición para el desarrollo infantil normal. Para Bowlby (1979), el tipo de apego que desarrollará el niño/a en relación a su cuidador principal dependerá de manera importante de la actitud y sensibilidad del adulto frente a las necesidades infantiles.

Así, desde el marco de la teoría del apego los estudios muestran que una adecuada respuesta sensible del cuidador se asocia positivamente con el desarrollo de un apego seguro en el niño/a (Bakermans-Kranenburg, van IJzerdoorn \& Juffer, 2003; van IJzerdoorn, 1995). En esta línea, investigaciones posteriores aportan evidencia sobre la relación entre sensibilidad, diferentes patrones de comportamiento de la madre y el estilo de apego desarrollado por el niño/a (Belsky \& Fearon, 2008; Kersten-Alvarez, Hosman, Riksen-Walraven, Van Doesum \& Hoefnagels, 2011; Meins, Fernyhough, Fradley \& Tuckey, 2001). También ha sido asociada al temperamento infantil (Kivijärvi, Räihä, Kaljonen, Tamminen \& Piha, 2005), a las conductas del bebé frente al dolor físico (Pillai Riddell et al., 2011), a la cantidad de llanto del infante frente al estrés (Lohaus, Keller, Ball, Voelker \& Elben, 2004) y a su desarrollo socioemocional (Page, Wilhelm, Gamble \& Card, 2010; Pearson et al., 2011). Estos antecedentes han llevado a proponer que la sensibilidad de la madre en las interacciones tempranas constituye un factor importante para predecir el desarrollo futuro del niño (Kemppinen, Kumpulainen, Raita-Hasu, Moilanen \& Ebeling, 2006).

Teniendo en cuenta el rol de la sensibilidad y su importancia en el desarrollo infantil, se han desarrollado numerosas investigaciones sobre el tema, las que han considerado principalmente factores como: la influencia del nivel socioeconómico y educacional de la madre, o su edad en esta competencia (Santelices et al., 2015), pero poco sabemos sobre factores contextuales como dificultades en el embarazo o parto. Considerando estos antecedentes, este estudio se focaliza en analizar cómo las dificultades perinatales pueden influir sobre la sensibilidad materna, buscando contribuir a la identificación de factores de riesgo que permitan apoyar e intervenir tempranamente en la díada madre-infante.

Sensibilidad materna y factores perinatales

La sensibilidad materna es definida por Bowlby (1979) como la respuesta del cuidador a las demandas in- fantiles, incluyendo la capacidad de leer e interpretar sus señales, respondiendo a ellas de manera apropiada. Ainsworth (1978), por su parte, considera que una madre sensible es aquella que es capaz de ponerse en el lugar de su bebé, y que está alerta a percibir e interpretar sus señales, respondiendo a ellas de manera apropiada e inmediata. Posteriormente, Crittenden (2005) la definió como un constructo diádico, que implica reciprocidad comunicativa. En este sentido, el adulto sensible estimula y tranquiliza al niño según lo vaya requiriendo, permitiéndole explorar e interactuar adecuadamente con su entorno. Beckwith, Chen y Hamilton (1999) por su parte, postulan que la sensibilidad puede ser predictora del desarrollo socio-emocional y cognitivo durante la infancia, los años preescolares, y la infancia tardía.

Distintos autores reportan una relación significativa entre los tipos de apego y el nivel de sensibilidad en las madres. Los estudios muestran patrones que se repiten entre tipos de apego infantil y sensibilidad parental; por ejemplo, padres responsivos y sensibles tienden a contribuir al desarrollo de apegos seguros en sus hijos/as, mientras que aquellos padres poco disponibles y con baja sensibilidad frente a las necesidades infantiles, tienden a contribuir al desarrollo de apegos ambivalentes e inseguros (Behrens, Parker \& Haltigan, 2011; Mills-Koonce et al., 2007). La literatura muestra, además, que el apego temprano repercute en el desarrollo socioemocional y la salud mental posterior del niño, siendo la sensibilidad parental un factor importante en ello (Ainsworth, Blehar, Waters \& Wall, 1978; Bolwby, 1979).

Sumado a la influencia de la sensibilidad parental en la construcción de los vínculos tempranos, algunos estudios sugieren que la mentalización, entendida como la capacidad de significar las experiencias propias y ajenas por medio de estados subjetivos y procesos mentales, predice la calidad del apego entre el niño y su cuidador (Fonagy et al., 2007). Esto se fundamenta en que la mentalización ejerce un rol fundamental en la cognición y regulación emocional del menor, quien logra alcanzar el entendimiento de sí mismo y de los demás, sólo luego de que ha sido comprendido por sus propios cuidadores (Fonagy et al., 2007, en Besoain \& Santelices, 2009). En esta línea, algunos estudios muestran que la función reflexiva de los cuidadores, relacionada con la mentalización, permite a los padres reflexionar sobre su propio apego y el de sus hijos, fomentando así el apego seguro (Slade et al., 2005, en Besoain \& Santelices, 2009). Aquellas madres con una función reflexiva parental adecuada, logran distinguir entre sus propios afectos y los de su hijo, siendo capaces de manejar los afectos negativos de su bebé a partir de la regulación de sus propias emociones (Grienenberger et al., 2005, en Besoain \& Santelices, 2009). 
Los estudios que exploran la relación entre sensibilidad materna y factores pre y perinatales reportan que las madres que no presentaron problemas de salud durante el embarazo, y que dieron a luz a través de parto natural, son más sensibles en la interacción con sus hijos/as (Bernier, Jarry-Boileau, Tarabulsy \& Miljkovitch, 2010). Otros estudios proponen que los eventos fisiológicos del embarazo, como el proceso de cesárea o parto normal, no afectan de forma directa la sensibilidad materna, pero sí pueden influir en el funcionamiento hormonal, generando inestabilidad emocional durante el período perinatal, interviniendo así en las interacciones tempranas madre-hijo/a (Fleming \& Corter, 1988).

Fleming y Corter (1988) se refieren a los factores fisiológicos vinculados al tipo de parto, y consideran que el flujo hormonal involucrado, participa en el moldeamiento y formación de la relación madre-hijo, incorporando la influencia de la organización biológica en los aspectos psicológicos. Estos estudios, sumados a los de Golberg (1983), muestran que las experiencias vividas durante el embarazo y el tipo de parto, puede influir en la calidad de las interacciones tempranas madre-hijo/a y si esto es así, es posible que esta y otras complicaciones biológicas vividas durante la etapa perinatal, ejerzan alguna influencia sobre la sensibilidad materna (Fleming, 1990). De lo anterior, se desprende que el parto involucra distintos cambios en la mujer a nivel emocional y biológico, los que pueden generar cierto grado de desestabilización para desarrollar en la madre una nueva organización, que le permita restablecer el equilibrio. Cualquier imprevisto o problema que ocurra en momentos cercanos al parto, puede generar una elevada carga afectiva la que podría afectar a la mujer psicológicamente, así como su capacidad para responder sensiblemente frente a las necesidades de su hijo/a (Fleming, 1990).

Como se mencionaba anteriormente, la experiencia del parto parece ser significativa en la calidad de la relación madre-hijo/a que se desarrolla luego del nacimiento. Bernier et al. (2010), intentan demostrar esto ejemplificando con casos de anemia durante el embarazo y postparto. Esta enfermedad puede causar una fuerte sensación de agotamiento físico, que cuando se combina con la constante demanda de atención y respuesta al niño/a por parte de la madre, puede interferir en su recuperación física y dejarla demasiado cansada como para responder adecuadamente a las necesidades del bebé. Así, la respuesta sensible puede verse negativamente afectada por la presencia de problemas físicos durante el embarazo o el parto, debido a su impacto en la madre y a su necesidad de recuperación, los que podrían lesionar sus competencias para interactuar positivamente con el bebé. Además de la recuperación física, podrían coexistir factores de estrés en la madre en cuanto al cuidado del niño/a, los que podrían generar sobreprotección o rechazo y gatillar actitudes maternales negativas en la interacción con el bebé (Bernier et al., 2010).

Los antecedentes teóricos presentados invitan a investigar la influencia de las complicaciones perinatales en las interacciones tempranas madre-bebé en mayor profundidad, de modo de pesquisar situaciones de riesgo para el desarrollo de una sensibilidad materna adecuada, así como para identificar la necesidad de intervenciones específicas para este grupo.

En base a lo anterior, el presente estudio tiene como objetivo general describir y analizar la influencia de la presencia de complicaciones perinatales, en la respuesta sensible de un grupo de madres chilenas hacia sus hijos/as entre diez y catorce meses, identificando efectos diferenciales según el tipo de complicación. Además, el nivel socioeconómico de las madres se toma en cuenta como una variable de control relevante, ya que numerosos estudios muestran una asociación entre este factor y la respuesta sensible del adulto (Farkas et al., 2015; Santelices et al., 2015).

\section{Método}

\section{Diseño}

Este estudio utiliza una metodología cuantitativa, y un diseño descriptivo, transversal, correlacional y explicativo. La variable dependiente considerada, es la sensibilidad de las madres hacia sus hijos/as, y las variables independientes son antecedentes pre y perinatales de la madre y del lactante. El nivel socioeconómico (NSE) se consideró como una variable a controlar en los análisis.

\section{Participantes}

La muestra estuvo compuesta por 90 diadas madre-hijo, residentes en la ciudad de Santiago de Chile, las que en un $56.7 \%$ se ubicaba en un NSE bajo, un $23.3 \%$ en un NSE medio y el $20 \%$ restante en un NSE alto. De los niños un $56.7 \%$ eran varones y un $43.3 \%$ eran niñas, todos asistían a salas cuna públicas o privadas, y sus edades se ubicaban entre 10 y 14 meses $(M=12.02$, $D S=1.298$ ).

\section{Instrumentos}

Escala de Sensibilidad en Adultos (E.S.A): Dicha escala fue creada en Chile por Santelices et al. (2012), definiendo la sensibilidad como la contingencia en responder a las señales y comunicaciones del niño/a a modo de favorecer su interés y cooperación en el juego, en un clima de afecto positivo. La escala se basa en la evaluación de 19 ítems (puntajes de 1 a 3) de la calidad de la relación madre-hijo/a, en base a la filmación de interacciones de 5 minutos de juego libre entre ambos miembros de la díada. A partir del puntaje asignado a cada ítem, se obtuvieron tres escalas que miden 
diferentes aspectos que inciden en la sensibilidad de las madres, las cuales también se ubican entre 1 y 3 puntos. La primera escala trata de la respuesta empática que indica qué tan capaz es el adulto de reconocer las señales del niño y responder a ellas interpretándolas adecuadamente. La segunda escala se refiere a la interacción lúdica, que evalúa la capacidad del adulto de relacionarse con el niño, tomando en cuenta sus iniciativas e incorporándose en el juego. Y por último, la tercera escala, referente a la expresión emocional, evalúa si el adulto manifiesta una actitud sensible y cálida frente a las necesidades y emociones del niño. $A$ partir del promedio de los puntajes obtenidos en estas tres escalas, se obtiene un puntaje total en sensibilidad, el cual se ubica en tres categorías: bajo, adecuado y alto. El primero de ellos, alude a un adulto que presenta dificultades para captar las señales del niño y a una interacción con el menor en donde prima un clima de afecto negativo. El nivel "adecuado" se refiere a un adulto que en ocasiones puede captar las señales del niño y responder apropiadamente a ellas, aunque no ocurre todo el tiempo. Por último, la sensibilidad alta, alude a un adulto que generalmente capta las señales del niño, responde a ellas de manera oportuna y adecuada, y que promueve un clima positivo y reforzador con el niño/a. La confiabilidad de la escala en una muestra similar a la de este estudio fue de 0.93, medida con Alfa de Cronbach.

Cuestionario sociodemográfico: Construido para los propósitos de este estudio, está compuesto de variadas preguntas que exploran datos del niño, la familia y las educadoras de la sala cuna a la que asisten. Para este estudio se consideró la información relativa al sexo y edad del niño en meses, y la presencia de complicaciones perinatales. Para explorar esta variable se consultó por: (a) presencia o ausencia de dificultades en el embarazo en la madre (variable dicotómica), (b) presencia o ausencia de dificultades en el embarazo en el niño (variable dicotómica), (c) presencia o ausencia de problemas en el parto (variable dicotómica), (d) tipo de parto (donde la cesárea no programada fue considerada como una dificultad), (e) peso al nacer (inferior a 2.500 grs. o superior a 4.000 grs., es decir, bajo o sobrepeso, según Organización Mundial de la Salud se consideró como complicación perinatal), y (f) edad gestacional en semanas (edad inferior a las 37 semanas se consideró problemática). Finalmente, se consideró la presencia de complicaciones perinatales en aquellas diadas con al menos una de las dificultades antes descritas, y un puntaje total que da cuenta de la cantidad de dificultades reportadas (rango de puntaje entre 0 y 6 , donde un mayor puntaje indica un mayor número de reportes).

\section{Procedimiento}

El proceso de recolección de datos se llevó a cabo mediante la aplicación del cuestionario sociodemográfico a las madres de los niños de doce meses. El contacto con los participantes se realizó mediante anuncios en distintas salas cuna de la capital, tras los cuales las madres interesadas en participar fueron contactadas e informadas acerca de la investigación y su objetivo, para finalmente firmar un consentimiento informado de participación en el estudio. El cuestionario sociodemográfico fue entregado para rellenar en sus casas a las participantes, al momento de aceptar su participación, y fue devuelto por las mismas a los investigadores al momento de realizar la filmación de la díada en la situación de juego libre, la cual fue posteriormente analizada por un codificador previamente capacitado. Las entrevistas así como la filmación de juego libre, se llevaron a cabo en la sala cuna a la cual asistía el niño o niña.

En el procedimiento se veló por los resguardos éticos y la confidencialidad, especificando cautelosamente en el consentimiento informado toda la información acerca de la investigación, los costos y beneficios de la participación, el tiempo requerido y los objetivos del estudio.

La codificación del juego libre de la díada con la E.S.A. se realizó por un equipo independiente de cuatro codificadoras, las que fueron entrenadas previamente en el uso del instrumento, hasta que lograron la confiabilidad deseada (al menos $80 \%$ de acuerdo con el Master Code generado por las autoras del instrumento). La confiabilidad del equipo se estimó en base al Kappa de Cohen, ubicándose en un rango entre .48 y .82.

\section{Análisis de resultados}

Primero, se evaluó la presencia de valores atípicos y datos perdidos presentes en los datos, como también el cumplimiento de los supuestos en las pruebas estadísticas realizadas. El criterio de significación utilizado fue $a=0.05$. Luego se analizaron los estadísticos descriptivos de las variables en estudio (análisis de frecuencia) y se realizaron análisis correlacionales (Spearman), comparativos (ANOVA) y de regresión, para cumplir con los objetivos del estudio.

\section{Resultados}

\section{Estadísticos descriptivos}

Los análisis muestran que un $26.7 \%$ ( 24 casos) de las madres de la muestra presentan una sensibilidad baja, mientras que un $56.7 \%$ (51 casos) de ellas obtienen una sensibilidad adecuada y un $16.7 \%$ (15 casos) una sensibilidad alta (ver Figura 1). Las madres evaluadas obtuvieron una media de $2.07(D . S .=0.398 ; \mathrm{N}=90)$ en su sensibilidad.

En relación a las variables pre y perinatales, un $21.1 \%$ (19 casos) de las madres reporta complicaciones durante el embarazo en ella y un 3.3\% (3 casos) en el niño/a en gestación. Un $15.6 \%$ (14 casos) reporta com- 
plicaciones en el parto y un $15.6 \%$ (14 casos) de los partos fueron por cesáreas no programadas. Un 14.4\% (13 casos) de los niños/a no alcanza la edad gestacional definida como adecuada y un $17.8 \% 16$ casos) no logra un peso adecuado al nacer. Así, un 48.9\% (44 casos) de la diadas reportan al menos una de las complicaciones perinatales anteriormente descritas, donde la mayoría refiere entre una y dos complicaciones (ver Figura 2). La media en complicaciones perinatales de las madres fue de $0.86(D . S .=1.076$, rango $0-4 ; \mathrm{N}=90)$.

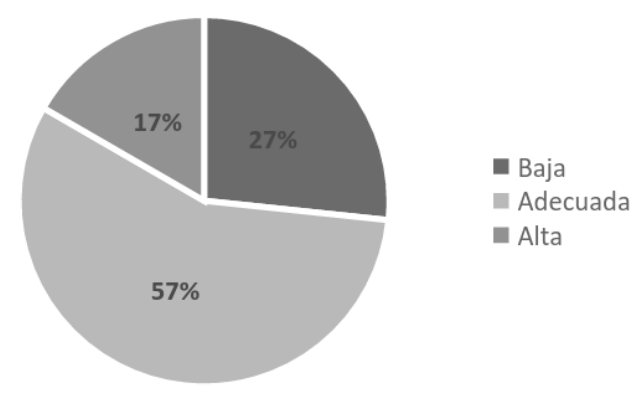

Figura 1. Porcentaje de madres según cada categoría de sensibilidad $(N=90)$

Influencia de las complicaciones perinatales sobre la sensibilidad de las madres hacia sus hijos/as de 12 meses

Se realizaron análisis con correlaciones de Pearson entre el puntaje obtenido en la categoría de complicaciones perinatales y la puntuación total, además de los puntajes de las escalas de sensibilidad, encontrándose que estas no eran significativas. Lo mismo se observó al realizar correlaciones parciales, controlando el NSE.

Luego se realizaron análisis comparativos con ANOVA entre los grupos de diadas con o sin complicaciones en total, así como en cada uno de los 6 aspectos considerados para la sensibilidad materna. No se observan diferencias significativas, no obstante, se observa una tendencia a que las madres que no presentaron complicaciones en su embarazo tengan una mayor sensibilidad, medida al año de edad de su hijos ( $F=3.042, p$ $=.085)$. Asimismo, su sensibilidad, aunque no de manera significativa, tendía a ser mayor si se presentaban complicaciones al momento del parto $(F=3.206, p=$ .077). Específicamente, se apreciaba una mayor interacción lúdica al año de edad ( $F=5.037, p=.027)$. Un último análisis, mostró una expresión emocional más sensible en las madres cuando el niño tenía una edad gestacional adecuada $(F=4.312, p=.041)$. No se observaron diferencias en relación a complicaciones del niño durante el embarazo, complicaciones por el tipo de parto, peso al nacer, o presencia/ausencia total de complicaciones.

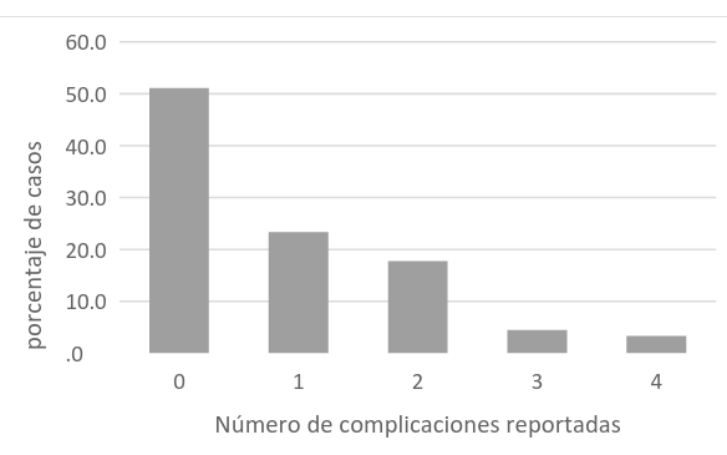

Figura 2. Porcentaje de casos según cantidad de complicaciones perinatales reportadas

Influencia del Nivel Socioeconómico sobre la sensibilidad que tienen las madres hacia sus hijos/as de 12 meses, así como sobre las complicaciones perinatales

Se realizó un ANOVA para evaluar si el Nivel Socioeconómico (NSE) influía en la sensibilidad de las madres (ver estadísticos descriptivos en Tabla 1). Se encontraron diferencias significativas en sensibilidad según NSE ( $F=20.226, p=.000)$, donde el NSE bajo obtuvo un menor puntaje en sensibilidad, respecto al NSE medio $(D S M=-.292, \mathrm{p}=.001)$ y NSE alto $(D S M=-.558$, $p=.000)$, seguido por el NSE medio, el cual obtuvo puntajes más bajos que el NSE alto $(D S M=-.266, p=$ .015). La Figura 3 muestra estos resultados.

Tabla 1. Estadísticos descriptivos de la sensibilidad materna según NSE

\begin{tabular}{llllll}
\hline & N & Mínimo & Máximo & Media & DS \\
\hline NSE Bajo & 51 & 1.31 & 2.68 & 1.8874 & .34133 \\
NSE Medio & 21 & 1.62 & 2.79 & 2.1789 & .37232 \\
NSE Alto & 18 & 1.98 & 2.79 & 2.4452 & .24759 \\
\hline
\end{tabular}

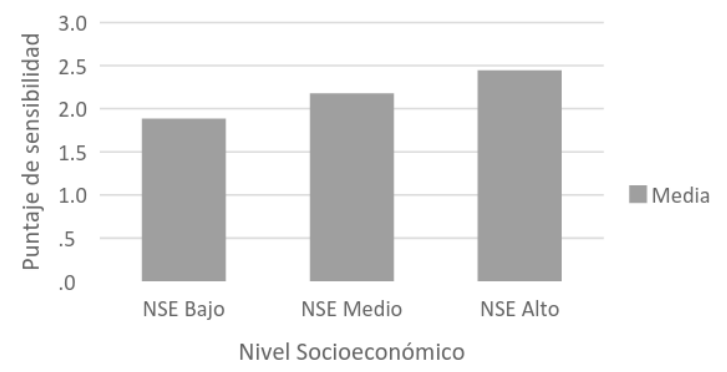

Figura 3. Distribución del puntaje de sensibilidad según Nivel Socioeconómico

No se observaron diferencias significativas según NSE en las complicaciones perinatales reportadas en los análisis de ANOVA, considerando el puntaje en complicaciones perinatales (rango 0 a 6), así como en los 
análisis con chi cuadrado para NSE y presencia/ausencia de complicaciones (ver estadísticos descriptivos y de frecuencia en Tablas 2 y 3 ).

Tabla 2. Estadísticos descriptivos de la cantidad de complicaciones perinatales según NSE

\begin{tabular}{llllll}
\hline & N & Mínimo & Máximo & Media & DS \\
\hline NSE Bajo & 51 & 0 & 4 & .84 & 1.155 \\
NSE Medio & 21 & 0 & 3 & 1.05 & .921 \\
NSE Alto & 18 & 0 & 4 & .67 & 1.029 \\
\hline
\end{tabular}

Tabla 3. Estadísticos de frecuencia de tipo de complicaciones perinatales según NSE

\begin{tabular}{lccc}
\hline & $\begin{array}{r}\text { NSE } \\
\text { Bajo }\end{array}$ & $\begin{array}{c}\text { NSE } \\
\text { Medio }\end{array}$ & $\begin{array}{c}\text { NSE } \\
\text { Alto }\end{array}$ \\
\hline $\begin{array}{l}\text { Presencia de complicaciones } \\
\text { en el embarazo en la madre }\end{array}$ & $23.5 \%$ & $19.0 \%$ & $16.7 \%$ \\
$\begin{array}{l}\text { Presencia de complicaciones } \\
\text { en el embarazo en el niño }\end{array}$ & $5.9 \%$ & - & - \\
$\begin{array}{l}\text { Presencia de complicaciones } \\
\text { en el parto }\end{array}$ & $11.8 \%$ & $19.0 \%$ & $11.1 \%$ \\
$\begin{array}{l}\text { Presencia de complicaciones } \\
\text { por tipo de parto }\end{array}$ & $9.8 \%$ & $28.6 \%$ & $16.7 \%$ \\
$\begin{array}{l}\text { Presencia de complicaciones } \\
\text { por peso al nacer }\end{array}$ & $13.7 \%$ & $19.0 \%$ & $11.1 \%$ \\
$\begin{array}{l}\text { Presencia de complicaciones } \\
\text { por edad gestacional }\end{array}$ & $19.6 \%$ & $19.0 \%$ & $11.1 \%$ \\
\hline
\end{tabular}

Influencia del NSE y complicaciones pre y perinatales sobre la sensibilidad de las madres

Se realizó una regresión jerárquica para analizar las variables que predecían la sensibilidad materna a los 12 meses de edad de los niños estudiados. Primero se ingresó el NSE de modo de controlar su efecto, observándose que era un predictor significativo $(B$ $=.563, t=6.394, p=.000)$, que explicó un $31.7 \%$ de la varianza en sensibilidad materna (ver Modelo 1 en
Tabla 1). Posteriormente, se probaron las distintas variables medidas para establecer complicaciones perinatales, resultando que los aspectos significativos para explicar sensibilidad eran presencia de complicaciones en el embarazo en la madre $(\beta=-163, t=-1.953, p=$ .050) y presencia de complicaciones en el parto $(\beta=$ $.231, t=2.311, p=.023$ ), aportando en conjunto un $6 \%$ de varianza adicional, siendo significativo $(p=.020)$ (ver Modelo 2 en Tabla 4). El modelo final propuesto explica un $37.7 \%$ de la varianza e indica que la sensibilidad materna entre los 10 y 14 meses de los niños será mayor en madres provenientes de NSE más altos, con ausencia de complicaciones durante su embarazo y presencia de complicaciones durante el parto. Estos resultados se discuten a continuación.

\section{Discusión}

El estudio desarrollado tuvo como objetivo analizar la influencia de las complicaciones perinatales tanto del niño como de la madre en la sensibilidad materna; considerando que la sensibilidad puede ser predictora de la calidad de la vinculación temprana y el desarrollo infantil, afectando los años preescolares y la infancia tardía (Beckwith et al., 1999; Bowlby, 1979). Los resultados, aunque distintos a lo esperado, muestran que algunas complicaciones perinatales sí influyen en la sensibilidad materna, y que su tipo de relación depende del tipo de complicación. Así, la ausencia de complicaciones en el embarazo en la madre se asocia con una mayor sensibilidad de ellas hacia sus hijos/as, mientras que la presencia de complicaciones durante el parto se asocia con una mayor sensibilidad materna hacia el año de edad de los niños/as.

Es necesario recordar que los eventos fisiológicos del embarazo, influyen en el funcionamiento hormonal, lo que puede generar desestabilidad emocional en la madre alrededor del nacimiento, interviniendo de esta forma, en la calidad de las interacciones de la diada (Fleming \& Corter, 1988). Estos resultados confirman parcialmente la hipótesis planteada al inicio del estudio, e invitan a profundizar en el tema, considerando

Tabla 4. Análisis de regresión jerárquica para sensibilidad materna a los 12 meses, con predictores de complicaciones pre y perinatales

\begin{tabular}{|c|c|c|c|c|c|c|}
\hline & \multicolumn{3}{|c|}{ Modelo 1} & \multicolumn{3}{|c|}{ Modelo 2} \\
\hline & B & SE B & B & B & SE B & B \\
\hline Intersección & 1.609 & .080 & & & & \\
\hline NSE & .281 & .044 & $.563^{* *}$ & .273 & .043 & $.548^{* *}$ \\
\hline Complicaciones en el embarazo en la madre & & & & -.163 & .083 & $-.168^{*}$ \\
\hline Complicaciones durante el parto & & & & .231 & .100 & $.198^{*}$ \\
\hline $\mathrm{R}^{2}$ & & 0.317 & & & 0.377 & \\
\hline F para el cambio en $\mathrm{R}^{2}$ & & $0.885^{* *}$ & & & $17.321^{* *}$ & \\
\hline
\end{tabular}

Nota. ${ }^{*} p<0.05 * * p<0.01$ 
el distinto rol y significado que dichas complicaciones pueden tener para la madre. Este hallazgo invita a prestar mayor atención al curso del embarazo, parto y postparto, ya que puede considerarse un predictor de la sensibilidad materna, clave en la configuración de un apego seguro.

Llama la atención especialmente, el que la presencia de complicaciones en el parto se asocie a un mayor nivel de sensibilidad en las madres. Frente a este resultado, se podría hipotetizar que la presencia de dificultades en el nacimiento, no así en el embarazo, llevan a las madres a estar más pendientes de las señales de sus hijos/as, impactando positivamente sobre su sensibilidad. Desde la perspectiva de la mentalización y función reflexiva (Fonagy et al., 2007, en Besoain \& Santelices, 2009), podría plantearse que madres con menor función reflexiva podrían tener más dificultades para manejar el embarazo y sus cambios, y reportar por tanto, mayor presencia de complicaciones durante el curso del embarazo en ellas mismas, mientras que las dificultades en el parto podrían activar la atención sobre el niño y su bienestar, estando las madres más pendientes de sus cuidados y desarrollo. Futuros estudios podrían aportar información más específica al respecto, considerando el tipo y la gravedad de dichas complicaciones, además de medidas para la función reflexiva materna.

El peso que tienen las complicaciones perinatales sobre la sensibilidad materna medida un año después de nacido el niño/a, es probablemente menor por el paso del tiempo, donde podrían sumarse otras variables de mayor peso no consideradas en este estudio. A pesar de lo anterior, resulta relevante el que algunas de estas dificultades, un año después, sigan teniendo un efecto. La variable que en este estudio obtuvo un peso más significativo fue el nivel socioeconómico, lo cual avala la importancia de considerarla y controlarla en estudios de este tipo, relacionándose directamente con la sensibilidad de las madres hacia sus hijos/as, lo que ya había sido reportado por otros estudios chilenos (Farkas et al., 2015; Santelices et al., 2015). Este resultado refleja una parte importante de las consecuencias de la desigualdad de oportunidades en la familia temprana chilena, asociadas a la pertenencia a un grupo con menores ingresos (Le Foulon \& Beyer, 2002), aspecto que ha orientado en los último años el desarrollo de políticas públicas para promover el apoyo y la igualdad de oportunidades para este grupo. Sumado a lo anterior, el sistema público de salud chileno presenta muchas falencias, entre ellas la inequidad de acceso (Mardones, 2004). Por otra parte, la baja calidad y amplia demora de atención médica en el sistema público, causa muchas veces tensión y descontento en quienes poseen menores ingresos y deben ser atendidos en el sistema público (Programa de las Naciones Unidas para el Desarrollo, 1998), lo cual produce mayor es- trés y desgaste. Estos podrían repercutir generando o aumentando el riesgo de posibles complicaciones en el embarazo y/o en el parto (Stein, Malmberg, Sylva, Barnes \& Leach, 2008), pudiendo ser esto un elemento contextual que afecta negativamente la sensibilidad en las madres más allá de la presencia o ausencia de complicaciones perinatales.

Estos resultados podrían asociarse a la función reflexiva parental, y su influencia sobre la calidad del apego (Besoain \& Santelices, 2009), infiriéndose que aquellas madres con un mayor NSE y probablemente con menores niveles de estrés, asociado al bienestar material, podrían presentar una mayor capacidad para reflexionar sobre sus propios estados afectivos y los de sus hijos, contribuyendo esto al despliegue de una mayor sensibilidad en el intercambio con ellos.

A pesar de que los hallazgos mencionados deben ser tomados con cautela por su carácter exploratorio, sugieren tomar conciencia sobre la influencia de las complicaciones pre y perinatales en la sensibilidad materna, de modo de pesquisar oportunamente a aquellas diadas que presentan un mayor riesgo, monitorear la evolución del vínculo con sus bebés e intervenir tempranamente en los casos que lo requieran.

Los presentes resultados son exploratorios y por ello sería interesante replicarlos en una muestra de mayor tamaño, considerando además población clínica. Se requiere además de nuevos estudios, idealmente longitudinales, que consideren edades más tempranas de los niños. El motivo de ello, se fundamenta en la posibilidad que las complicaciones perinatales tengan un mayor efecto al evaluar la sensibilidad materna posterior al parto, evento que podría disminuir su influencia en la medida en que el niño crece, pudiendo surgir otras variables que expliquen mejor el tipo de interacción que establece la madre con su hijo. Futuras investigaciones que incluyan otros factores (como por ejemplo el nivel de estrés en las madres, embarazos deseados versus no deseados, presencia y calidad de la red de apoyo, entre otros), podrían arrojar mayor información. En esta misma línea, resultaría beneficioso estudiar los lugares de atención en que se realizan los controles y atenciones perinatales y sus características, pudiendo esto explicar diferencias en el tipo de afrontamiento médico de las dificultades y el tipo de experiencia vivida por la madre. Estudios de mayor especificidad y con un mayor número de variables contextuales podrían aportar en la pesquisa oportuna de dificultades vinculares, en la prevención en salud mental infantil y en la implementación de intervenciones precoces para mejorar la sensibilidad materna.

\section{Referencias}

Ainsworth, M., Blehar, M., Waters, E. \& Wall, S. (1978). Patterns of attachment: A psychological study of 
the strange situation. Hillsdale: Lawrence Erlbaum Associates.

Bakermans-Kranenburg, M., van IJzendoorn, M. \& Juffer, F. (2003). Less is more: Meta-analyses of sensitivity and attachment interventions in early childhood. Psychological Bulletin, 129(2), 195-215. doi:10.1037/0033-2909.129.2.195

Beckwith, L., Chen, S. \& Hamilton, C. (1999). Maternal sensitivity during infancy and subsequent life events relate to attachment representation at early adulthood. Developmental Psychology, 35(3), 693-700. doi:10.1037/0012-1649.35.3.693

Behrens, K., Parker, A. \& Haltigan, J. (2011). Maternal sensitivity assessed during the strange situation procedure predicts child's attachment quality and reunion behaviors. Infant Behavior \& Development, 34(2), 378-381. doi:10.1016/j.infbeh.2011.02.007

Belsky, J. \& Fearon, P. (2008). Precursors of Attachment Security. En J. Cassidy \& P.. Shaver (Eds.). Handbook of attachment. Theory, research, and clinical applications (pp. 295-316). New York: The Guilford Press.

Bernier, A., Jarry-Boileau, V., Tarabulsy, G. \& Miljkovitch, R. (2010) Initiating a caregiving relationship: Pregnancy and childbirth factors as predictors of maternal sensitivity. Infancy, 15(2), 197-208. doi:10.1111/j.1532-7078.2009.00006.x

Besoain, C. \& Santelices, M. (2009). Transmisión intergeneracional del apego y función reflexiva materna: Una revisión. Terapia Psicológica, 27(1), 113-118. doi:10.4067/S0718-48082009000100011

Bowlby, J. (1969). El vínculo afectivo. Buenos Aires: Paidós.

Bowlby, J. (1979). Vínculos afectivos: Formación, desarrollo y pérdida. Madrid: Morata.

Crittenden, P. (2005). Care-Index para toddlers: Manual de Codificación. Miami: Family Relations Institute.

Crittenden, P. (2006). A dynamic maturational model of attachment. Australian and New Zealand Journal of Family Therapy, 27, 105-115. doi:10.1002/j.1467-8438.2006.tb00704.x

Farkas, C., Carvacho, C., Galleguillos, F., Montoya, F., León, F., Santelices, M. \& Himmel, E. (2015). Estudio comparativo de la sensibilidad entre madres y personal educativo en interacción con niños/as de un año de edad. Perfiles Educativos, 36(148), 16-33. Recuperado de http:// www.iisue.unam.mx/perfiles/perfiles articulo. php?clave $=2015-148-16-33$
Fleming, A. (1990). Hormonal and experiential correlates of maternal responsiveness in human mothers. En N. Krasnegor \& R. Bridges (Eds.), Mammalian parenting: Biochemical, neurobiological, and behavioral determinants (pp. 184208). New York: Oxford University Press.

Fleming, A. \& Corter, C. (1988). Factors influencing maternal responsiveness in humans: Usefulness of an animal model. Psychoneuroendocrinology, 13, 189-212. doi:10.1016/0306-4530(88)90014-5

Goldberg, S. (1983). Parent-infant bonding: Another look. Child Development, 54(6), 1355-1382. doi: $10.2307 / 1129800$

Kemppinen, K., Kumpulainen, K., Raita-Hasu, J., Moilanen, I. \& Ebeling, H. (2006). The continuity of maternal sensitivity from infancy to toddler age. Journal of Reproductive and Infant Psychology, 24, 199-212. doi:10.1080/02646830600821249

Kersten-Alvarez, L., Hosman, C., Riksen-Walraven, J., Van Doesum, K. \& Hoefnagels, C. (2011). Which preventive interventions effectively enhance depressed mother's sensitivity? A meta-analysis. Infant Mental Health Journal, 32, 362-376. doi:10.1002/imhj.20301

Kivijärvi, M., Räihä, H., Kaljonen, A., Tamminen, T. \& Piha, J. (2005). Infant temperament and maternal sensitivity behavior in the first year of life. Scandinavian Journal of Psychology, 46, 421428. doi:10.1111/j.1467-9450.2005.00473.x

Le Foulon, C. \& Beyer, H. (2002). Un recorrido por las desigualdades salariales en Chile. Estudios Públicos, 85, 139-175. Recuperado de http://www. cepchile.cl/1 3031/doc/un recorrido por las desigualdades salariales en chile.html\#.VX7EMmdFBIA

Lohaus, A., Keller, H., Ball, J., Voelker, S. \& Elben, C. (2004). Maternal sensitivity in interactions with three- and 12-month-old infants: Stability, structural composition, and developmental consequences. Infant and Child Development, 13, 235-252. doi:10.1002/icd.351

Mardones, F. (2004). Algunos antecedentes sobre la inequidad en la situación de salud del adulto mayor en Chile. Revista médica de Chile, 132(7), 865872. doi:10.4067/S0034-98872004000700013

Martínez, C. (2011). Mentalización en psicoterapia: Discusión sobre lo explícito e implícito de la relación terapéutica. Terapia Psicológica, 29(1), 97-105. doi:10.4067/S0718-48082011000100010

Meins, E., Fernyhough, C., Fradley, E., \& Tuckey, M. (2001). Rethinking maternal sensitivity: Mothers' comments on infants' mental processes 
predict security of attachment at 12 months. Journal of Child Psychology and Psychiatry, 42, 637-648. doi:10.1111/1469-7610.00759

Mills-Koonce, W., Gariépy, J., Propper, C., Sutton, K., Calkins, S., Moore, G. \& Cox, M. (2007). Infant and parent factors associated with early maternal sensitivity: A caregiver-attachment systems approach. Infant Behavior and Development, 30(1), 114-126. doi:10.1016/j.infbeh.2006.11.010

Organización Mundial de la Salud. (2003). Pregnancy, childbirth, postpartum and newborn care: a guide for essential practice. Ginebra: World Health Organization. Recuperado de http:// www.afro.who.int/index.php?option $=$ com docman\&task $=$ doc download\&gid $=2011$

Page, M., Wilhelm, M., Gamble, W. \& Card, N. (2010). A comparison of maternal sensitivity and verbal stimulation as unique predictors of infant social-emotional and cognitive development. Infant Behavior and Development, 33, 101-110. doi:10.1016/j.infbeh.2009.12.001

Pearson, R., Heron, J., Melotti, R., Joinson, C., Stein, A., Ramchandani, P \& Evans, J. (2011). The association between observed non-verbal maternal responses at 12 months and later infant development at 18 months and IQ at 4 years: A longitudinal study. Infant Behavior and Development, 34, 525-533. doi:10.1016/j.infbeh.2011.07.003

Pillai Riddell, R., Campbell, L., Flora, D., Racine, N., Din Osmun, L., Garfield, H. \& Greenberg, S. (2011). The relationship between caregiver sensitivity and infant pain behaviors across the first year of life. International Association for the
Study of Pain, 152, 2819-2826. doi:10.1037/00121649.32.1.12

Programa de las Naciones Unidas para el Desarrollo (1998). Informe Desarrollo Humano en Chile. Las Paradojas de la Modernización. Santiago: Autor.

Santelices, M., Carvacho, C., Farkas, C., León, F., Galleguillos, F. \& Himmel, E. (2012). Medición de la Sensibilidad del Adulto con Niños de 6 a 36 Meses de Edad: Construcción y Análisis Preliminares de la Escala de Sensibilidad del Adulto, E.S.A. Terapia psicológica, 30(3), 19-29. doi: 10.4067/S0718-48082012000300003

Santelices, M., Farkas, C., Montoya, M., Galleguillos, F., Carvacho, C., Fernández, A., Morales, L., Taboada, C. \& Himmel, E. (2015). Factores predictivos de sensibilidad materna en infancia temprana. Psicoperspectivas, 14(1), 66-76. doi:10.5027/ PSICOPERSPECTIVAS-VOL14ISSUE1-FULLTEXT-441

Stein, A., Malmberg, L., Sylva, K., Barnes, J. \& Leach, P. (2008). The influence of maternal depression, caregiving, and socioeconomic status in the post-natal year on children's language development. Child: Care, Health And Development, 34(5), 603-612. doi:10.1111/j.13652214.2008.00837.x

van IJzendoorn, M. (1995). Adult attachment representations, parental responsiveness, and infant attachment: A meta-analysis on the predictive validity of the adult attachment interview. Psychological Bulletin, 117(3), 387-403. doi:10.1037/0033-2909.117.3.387 